\title{
DESCRIÇÃO DE DANOS E VOLUME DE FITOMASSA LENHOSA DE FUSTES DE Cassia siamea LAM. SECCIONADOS POR Coccoderus novempunctatus (COLEOPTERA: CERAMBYCIDAE) ${ }^{1}$
}

Antônio José da Silva Neto², Henrique Trevisan ${ }^{3}$, Luiz Santana do Nascimento ${ }^{4}$ e Acacio Geraldo de Carvalho ${ }^{5}$

\begin{abstract}
RESUMO - Cerambicídeos que promovem a queda de galhos ou fustes podem causar danos a diversas espécies florestais, sejam em plantios econômicos ou mesmo em árvores utilizadas em arborização. O cerambicídeo Coccoderus novempunctatus é um destes agentes. O dano realizado pela larva propicia a queda de ramos de muitas espécies florestais e, no caso ora avaliado, em fustes de Cassia siamea ou parte destes. Esta é uma espécie florestal utilizada em arborização e recuperação de áreas perturbadas ecologicamente. O trabalho teve o objetivo de diagnosticar os danos de C. novempunctatus em C. siamea, através da diagnose das lesões e da quantificação do volume de madeira dos fustes por ele seccionados. Cinco parcelas foram demarcadas em mata secundária, onde a atuação do inseto havia sido registrada. Os fustes de C. siamea, cortados pelo inseto, tiveram seu diâmetro no ponto da secção mensurado bem como o comprimento total. Os volumes de madeira foram calculados utilizando-se o método de Smalian. O diâmetro médio no ponto da secção e o comprimento médio de fustes de $C$. siamea, cortados por $C$. novempunctatus foi 9,07 cm e 6,31 m, respectivamente. O volume médio de madeira, destes fustes, com casca e sem casca foi 0,0261 e 0,0218 $\mathrm{m}^{3}$, respectivamente.

Palavras chave: Leguminosae, Pragas florestais e Arborização.
\end{abstract}

\section{DESCRIPTION OF DAMAGES AND VOLUME OF WOOD PHYTOMASS OF Cassia siamea LAM STEMS CUT FOR Coccoderus novempunctatus (COLEOPTERA:CERAMBYCIDAE)}

\begin{abstract}
Cerambycids that promote breaking of branches or stems may cause damage to several forest species, in economical plantings or even in trees used in urban afforestation. Coccoderus novempunctatus (Coleoptera: Cerambycidae) is one of these cases. The damage accomplished by the larvae propitiates the fall branches of many forest species, and in the case evaluated in this study, can cause fall of Cassia siamea stems or part of these. Cassia siamea is a forest specie used in afforestation and in recovery of ecologically disturbed areas. Thus, the objective of this work was to evaluate the damages caused by C. novempunctatus on C. siamea through lesions diagnoses and volume quantification of wood of stems sectioned by it. Five plots were demarcated in a secondary forest where the insect action had been registered. C. siamea stems cut by insect had their diameter in the cut point measured as well as total length. Wood volumes were calculated by using the Smalian method. Average diameter in the cut point, and the average length of C. siamea stems cut by C. novempunctatus was $9.07 \mathrm{~cm}$ and $6.31 \mathrm{~m}$, respectively. Average wood volume of these stems with and without bark was 0.0261 and $0.0218 \mathrm{~m}^{3}$, respectively.
\end{abstract}

Keywords: Afforestation, Forest pests and Leguminosae.

\footnotetext{
${ }^{1}$ Recebido em 18.06.2008 e aceito para publicação em 20.04.2011.

${ }^{2}$ Programa de Pós-graduação em Engenharia Florestal Pela Universidade Federal de Lavras. E-mail: <antoniojsnd@yahoo.com.br>. ${ }^{3}$ Doutorado em Ciências Ambientais e Florestais, Universidade Federal Rural do Rio de Janeiro. E-mail: <hentrevisan@gmail.com>.

${ }^{4}$ Mestrado em Ciências Ambientais e Florestais, Universidade Federal Rural do Rio de Janeiro. E-mail: <lunassan@yahoo.com.br >.

${ }^{5}$ Universidade Federal Rural do Rio de Janeiro, Instituto de Florestas, Departamento de produtos Florestais. E-mail: <acacio@ufrrj.br>.
} 


\section{INTRODUÇÃO}

O dano de pragas às essências florestais é uma questão que merece atenção em qualquer atividade no setor florestal. Um grande número de insetos pode causar prejuízos na madeira, nas folhas e nas sementes. Segundo Flechtmann (1995), insetos degradadores de madeira são, potencialmente, limitantes para o desenvolvimento, o crescimento e a reprodução das árvores, pois, além de provocarem danos em diferentes partes, podem ser vetores de doenças, bactérias, fungos e vírus.

Dentro da classe insecta, os coleópteros merecem destaque por assumirem grande importância ecológica e econômica. A família Cerambycideae, pertencente à ordem Coleoptera, abriga um grande número de insetos reconhecidamente danosos a diversas espécies florestais, pois suas larvas ou o inseto adulto podem causar a morte ou o depauperamento da planta.

O coleóptero Coccoderus novempunctatus, segundo Monné (2005), ocorre nos estados de Goiás, Mato Grosso, Distrito Federal, Rio Grande do Norte, Rio Grande do Sul, no Paraguai, na Argentina (nas localidades de Misiones, Salta, Santiago Del Estero, Chaco, Entre Rios e Buenos Aires) e também no Uruguai. Ocorre praticamente em toda a extensão da Mata Atlântica e ainda nas florestas tropicais do Brasil central. Como relatou Bondar (1915), os estragos produzidos por este inseto em várias leguminosas de São Paulo e do Rio de Janeiro são semelhantes aos produzidos pelo Diploschema rotundicolle (Coleoptera: Cerambycidae) nas laranjeiras.

Segundo Monné (2001), as seguintes plantas foram citadas como hospedeiras para C. novempunctatus: Caesalpinia echinata, C. peltophoroides, Carpotroche brasiliensis, Cassia ferruginea, C. fistula, C. grandis, C.macranthera, C. nodosa, C. reticulata, C.strobilacea, Delonix regia, Tamarindus indica (Caesalpiniaceae), Acacia dealbata, A. decurrens, A. decurrens mollissima, A. praecox, Enterolobium contortisiliquum, Inga affinis, I. sessilis, I. uruguensis, I. vera, Mimosa detinens, Piptadenia communis, (Mimosaceae) e Blepharistemma tweediei (Myrtaceae). Di Iorio (1998) incluiu novas informações sobre plantas hospedeiras, biologia e descreveu a arquitetura das galerias das larvas em quatro plantas hospedeiras.

Silva Neto et al. (2008) registraram a ocorrência de $C$. novempunctatus causando danos em Cassia siamea, leguminosa muito empregada na arborização de vias urbanas e utilizada na recuperação de áreas degradadas. C. siamea pode ser encontrada em regiões secas e semiáridas da Ásia e África. No nordeste do Brasil, esta árvore é encontrada facilmente, pois cresce bem em climas de monções ou estações úmidas, subúmidas e semiáridas (FARIDAH; VAN DER MAESEN, 1997).

Trabalhos que avaliam o volume de madeira envolvido com o ciclo de vida de cerambicídeos associados à degradação deste material são escassos, sendo, portanto, um parâmetro importante na avaliação do estudo de aspectos tróficos desse grupo de insetos, bem como dos danos por eles causados em espécies florestais. No aspecto danos, Zanuncio et al. (2005) evidenciam que coleópteros broqueadores vêm assumindo maior importância, comparados a outras pragas florestais. Esse grupo de insetos suscita também discussões no impacto de sua atuação no processo de deterioração da madeira. Nesse sentido, Trevisan et al. (2007) enfatizam que o estudo deste caráter deve envolver áreas distintas do conhecimento, como a entomologia, e que o entendimento integral do processo de deterioração engloba aspectos ecológicos associados aos organismos xilófagos ou mesmo à madeira estudada.

Nesse sentido, este trabalho teve como objetivo descrever os danos realizados por C. novempunctatus em fustes de $C$. siamea, bem como quantificar o volume de madeira dos segmentos seccionados pelo inseto e registrar espécies arbóreas lesionadas pelo inseto na área do experimento.

\section{MATERIAL E MÉTODOS}

As observações dos danos causados por $C$. novempunctatus (Figura 1) em C. siamea foram realizadas na Floresta Nacional Mário Xavier, em Seropédica, RJ. Nesta localidade, ocorre um grupamento espontâneo dessa espécie florestal. Esta unidade de conservação está localizada na região bioclimática 4, segundo classificação de Golfari (1980), com altitude entre 0 e 800 m. A região apresenta inverno seco, com temperatura média anual de 18 a $24^{\circ} \mathrm{C}$, e precipitação média anual de 1.200 a $2.000 \mathrm{~mm}$; é caracterizada por déficit hídrico de 0 a 30 mm/ ano. As precipitações estão bem distribuídas, com predomínio no semestre mais quente.

Foram demarcadas cinco parcelas de 10x20 m e identificadas suas espécies arbóreas. As árvores visualmente atacadas foram marcadas com uma circunferência no local onde o inseto seccionou o fuste, 


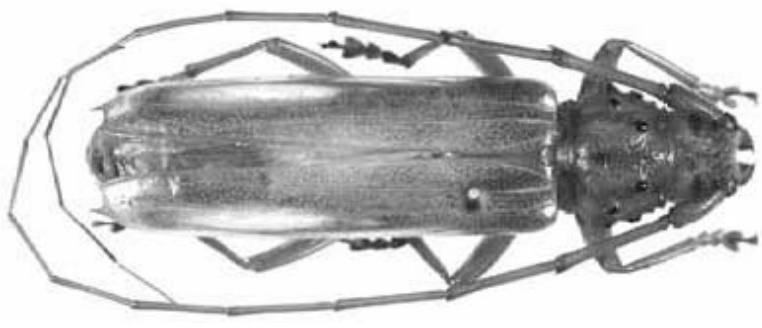

Figura 1 - Macho de Coccoderus novempunctatus com 27,5 mm de comprimento Monné (2005).

Figure 1-Coccoderus novempunctatus male with $27.5 \mathrm{~mm}$ length Monné (2005).

ou parte dele, que, em seguida, foi mensurada. As observações para a determinação do volume de madeira foram realizadas em oito fustes de $C$. siamea, seccionados por C. novempunctatus. A espessura da lesão, feita em espiral, que provoca a queda do fuste, também foi mensurada, bem como o comprimento da seção seccionada pelo inseto.

Para a determinação dos volumes dos fustes cortados, foram feitas as cubagens rigorosas deles, aplicando-se o método de Smalian, de acordo com Machado e Filho (2003). As seções cortadas, em função da atuação da larva, foram divididas em toras de $1 \mathrm{~m}$ de comprimento, sendo a última porção, cujo tamanho era inferior a $1 \mathrm{~m}$, considerada a ponta.

Foram mensuradas as circunferências nas duas extremidades destas seções e calculadas as respectivas áreas transversais. O volume das seções foi obtido através da aplicação da fórmula a seguir. Para a quantificação do volume total de fitomassa lenhosa derrubada por árvore, foram também cubados os galhos com circunferência superior ou igual a $9,5 \mathrm{~cm}$ na junção com o fuste, e seus volumes somados ao volume do segmento principal.

A coleta dos dados citados foi realizada utilizandose fita métrica e trena. As fórmulas aplicadas para os cálculos dos volumes são:

$V=\{[(g 1+g 2) / 2] \times L+[(g 2+g 3) / 2] \times L+\ldots+$ $[(g n-1+g n) / 2] \times L\}+V p$

$V=$ Volume do tronco com casca

$g 1$ = Área transversal na base da tora $=\mathrm{C}_{1}{ }^{2} / 4 ð$

$g 2$ = Área transversal no topo da tora $=\mathrm{C}_{2}{ }^{2} / 4$ ð

$L=$ Comprimento da tora
O volume da ponta ( $\mathrm{Vp}$ ) foi calculado através da fórmula: $\mathrm{Vp}=($ gn $x \mathrm{Lp}) / 3$, e gn e Lp correspondem à área basal do topo da última tora e Lp ao comprimento da ponta, respectivamente. O volume sem casca dos troncos foi obtido aplicando-se as mesmas fórmulas, porém considerando as áreas transversais sem casca.

A altura em relação ao solo, do local onde ocorreu a secção do fuste, foi mensurada, bem como o DAP (diâmetro a altura do peito) das árvores danificadas, com o auxílio de uma trena. Essas variáveis foram analisadas pela correlação de Pearson, sendo processadas no programa estatístico BioEstat 4.0.

As lesões foram fotografadas para ilustrar e auxiliar na diagnose dos danos, e esta foi caracterizada por uma descrição detalhada das lesões realizadas pelo inseto na planta.

\section{RESULTADOS E DISCUSSÃO}

Foram encontradas dez espécies arbóreas nas parcelas avaliadas, sendo estas: Caesalpinia ferrea Lam., Couroupita guianensis Aubl. , Lophantera lactescens Ducke,, Mangifera indica L., Mimosa bimucronata (DC.) Kuntze, Samanea saman (Jacq.) Merr., Tabebuia chrysotricha (Mart. ex DC.) Standl, Anadenanthera macrocarpa (Benth.), Cassia siamea (Lam.) e Cassia grandis L.f, sendo registrada a atuação do inseto somente nas três últimas.

Foram contabilizados 32 fustes de C. siamea. Em 18 deles foi registrado o dano do inseto, perfazendo 56\% dos indivíduos com lesão evidente. O volume de madeira de fustes derrubados pela ação do inseto foi obtido em 8 amostras, sendo este o número de seções de fustes encontrados no solo e consistindo, portanto, de $44 \%$ dos indivíduos.

O dano avaliado é caracterizado por um corte em espiral na região do alburno, que se estende formando uma galeria na região da medula do fuste. O galho ou ramo caiu em função da ampliação da lesão em espiral, sendo derrubado pelo ação do vento ou pelo próprio peso. A ruptura do fuste ocorre em função da atuação da larva do inseto na sua parte interna, atingindo a região da periferia do alburno. A espessura média de $C$. siamea, desta área onde

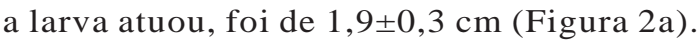

Revista Árvore, Viçosa-MG, v.35, n.4, p.801-807, 2011 


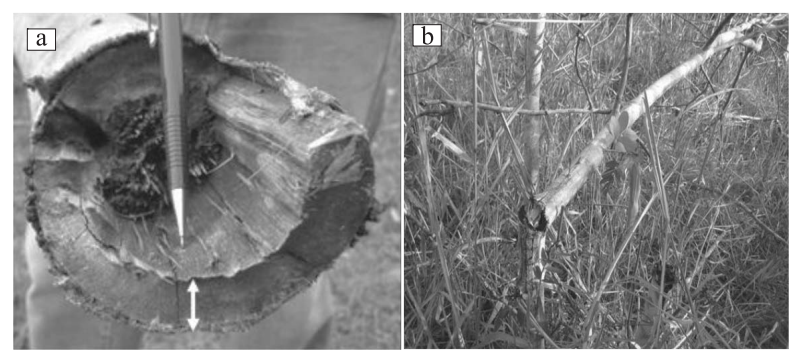

Figura 2 - (a)-Detalhe do corte em espiral, realizado pela larva de Coccoderus novempunctatus em fuste de Cassia siamea; (b)-fuste de Cassia siamea seccionado por Coccoderus novempunctatus. Seropédica, RJ, 2008.

Figure 2-(a) Cut spiral detail accomplished by Coccoderus novempunctatus larvae in Cassia siamea stem; (b) C. siamea Stem cut by C. novempunctatus. Seropédica, RJ, 2008.

Alguns cerambicídeos têm o hábito de cortar os galhos de seus hospedeiros para postura e consequentemente para o desenvolvimento das larvas. Com esse comportamento, os indivíduos do gênero Oncideres podem ser considerados os principais. Porém, neste caso, esta ação é promovida pelo adulto, que efetua o anelamento de galhos, e estes caem em função de seu peso ou da ação do vento, sendo este o sítio de desenvolvimento das larvas.

No caso do cerambicídeo C. novempunctatus, o inseto promove a queda do fuste inteiro ou parte dele, comportamento registrado em C. siamea (Figura 2b). Em outras espécies florestais, este cerambicídeo secciona ramos, como observado nesse experimento, em $A$. macrocarpa e C. grandis. A postura é realizada no fuste da árvore viva, pois todo o desenvolvimento larval ocorre nesta condição.

A queda do fuste é promovida por uma lesão em espiral, realizada pela larva do inseto (Figura 2), diferente de Oncideres sp., que promove uma lesão em forma de anel e preconizada pelo adulto.

Bondar (1915) observou, em D. regia, que a larva de $C$. novempunctatus se desenvolve em madeira viva, escavando uma longa galeria que desce dos ramos finos aos mais grossos até o tronco. Neste experimento, a galeria feita pela larva é realizada em espiral e em diversos planos, como registrado no fuste de $C$. siamea.

Silva e Almeida (1941) descreveram e ilustraram os estragos causados pela larva em Caesalpinea echinat, e observaram que ela conserva a galeria limpa, retirando toda a serragem através de orifícios na casca em diversas alturas ou distâncias. Ao atingir seu total desenvolvimento, deriva da direção geral axial em curvas que se aproximam da região cortical, onde prepara a câmara pupal, vedando a entrada com raspas de madeira, que é comprimida em massa compactada com o auxílio da serragem. O ciclo de vida foi estimado em aproximadamente dois anos.

Esses orifícios também foram observados em $C$. siamea e, neste caso, foi constatado que o vegetal reage a essa lesão, exsudando uma substância gomosa de cor escura, que escorre pelo fuste (Figura 3a), evidenciando a presença do inseto na planta.

Também foi observado que abaixo e em torno da ruptura, por consequência da lesão em espiral, a árvore emite brotações (Figura 3b), conferindo, portanto, um crescimento irregular à copa, afetando, dessa forma, a estética da árvore e, futuramente, quando os ramos se desenvolverem, a sua estabilidade.

A correlação entre a altura do ponto onde o inseto seccionou o fuste e o DAP deste revelou significância na relação dessas variáveis (Figura 4), indicando que quanto maior o DAP da árvore maior foi a altura do ponto onde a larva fez a lesão que ocasionou a ruptura de parte do fuste. A explicação pode estar associada ao fato de a lesão estar em um local onde o diâmetro é superior, ou seja, onde o fuste é mais grosso.

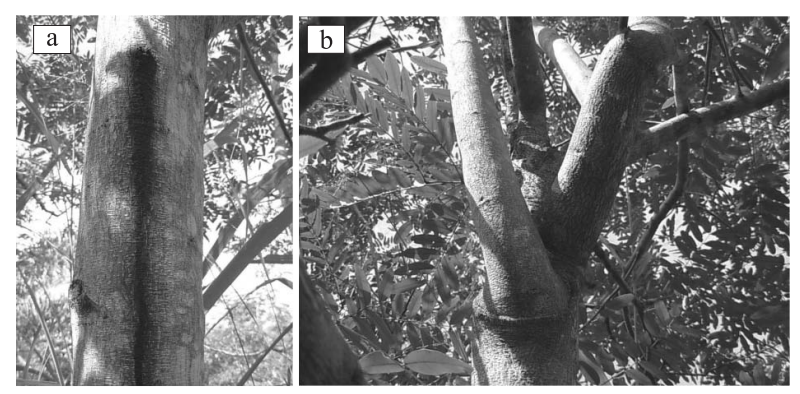

Figura 3 - (a)-Exsudado de Cassia siamea em função de orifício construído pela larva; (b)- Brotações emitidas devido a ruptura do fuste, ocasionada pela atuação da larva de Coccoderus novempunctatus. Seropédica, RJ, 2008.

Figure 3-(a)-Cassia siamea ooze according of the built hole by larvae (b) - Shoots issued due stem disruption caused by Coccoderus. novempunctatus larvae action. Seropédica, RJ, 2008. 


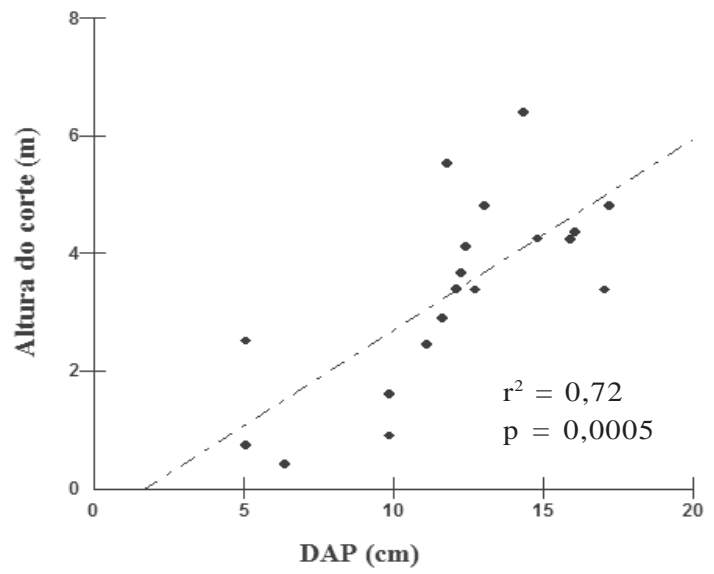

Figura 4 - Correlação de Pearson para as variáveis diâmetro à altura do peito (DAP) e à altura do corte de fustes de Cassia siamea seccionados por Coccoderus. novempunctatus. Seropédica, RJ,2008.

Figure 4-Pearson's correlation for variables diameter at breast height (DBH) and cut height for Cassia siamea stems split up by Coccoderus novempunctatus. Seropédica, RJ,2008.

Nesse sentido, foi observado que, em alguns fustes lesionados que não caíram, houve, uma reação fisiológica no sentido de sanar o dano, caracterizada por espessamento da casca, que confere um aspecto saliente ao local, no qual o exsudado também pode ocorrer (Figura 5). Nesses fustes, foi observado que a ruptura ocorreu a uma altura superior.

O diâmetro médio no ponto da secção dos fustes de $C$. siamea foi de $9,07 \mathrm{~cm}$, variando de 7,32 a 11,14 cm (Tabela 1). O comprimento médio das seções dos fustes seccionados foi $6,31 \mathrm{~m}$, sendo registrada uma variação de 4,70 a 9,45 m (Tabela 1 ).

O volume médio da madeira com casca (VC) e sem casca (VSC) de fustes de $C$. siamea seccionados por C. novempunctatus foi de $0,0261 \mathrm{~m}^{3}$ e $0,0218 \mathrm{~m}^{3}$, respectivamente (Tabela 2). O percentual médio de casca dos fustes cortados pelo inseto foi de $16,46 \%$ (Tabela 2). Costa (1986), estudando o volume médio de material lenhoso seccionado por Oncideres impluviata, na espécie florestal Mimosa scabrella Benth, obteve o valor de $102,593 \mathrm{~cm}^{3}\left(0,000102593 \mathrm{~m}^{3}\right)$.

No sentido de comparar a ação de dois cerambicídeos que possuem o mesmo hábito, ou seja, o de seccionar ramos ou fustes, utilizados nos seus ciclos de vida, pode-se dizer que o volume médio de

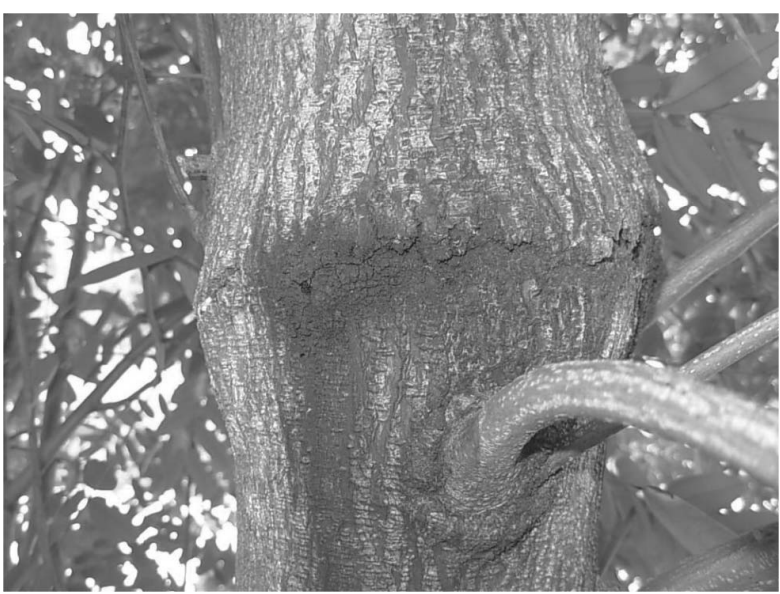

Figura 5 - Aspecto saliente do fuste de Cassia siamea, em função da reação fisiológica da planta devido a lesão em espiral provocada pela larva de Coccoderus novempunctatus. Seropédica, RJ, 2008.

Figure 5 - Salient aspect of Cassia siamea stem, according to the plant physiological reaction due spiral lesion caused by the Coccoderus novempunctatus larvae. Seropédica, RJ, 2008.

material lenhoso registrado por Costa (1986) não chega a 0,5\% do volume médio registrado para o cerambicídeo aqui avaliado, evidenciando, dessa forma, uma ação bem mais nociva à espécie florestal utilizada como hospedeira por $C$. novempunctatus. Para uma pesquisa melhor desse aspecto, seria necessária uma análise onde outros parâmetros, como biologia e o potencial biótico dos insetos, fossem levados em consideração, o que não é o caso da abordagem deste trabalho.

Tabela 1-Diâmetro no ponto de secção (DPS e comprimento de fustes de Cassia siamea seccionados por Coccoderus novempunctatus. Seropédica, RJ. 2008.

Table 1 - Diameter at the section point (DSP) and length of Cassia siamea stems cut by Coccoderus novempunctatus. Seropédica, RJ. 2008.

\begin{tabular}{ccc}
\hline Fuste & DPS $(\mathrm{cm})$ & Comprimento $(\mathrm{m})$ \\
\hline 1 & 8,44 & 4,90 \\
2 & 9,23 & 6,80 \\
3 & 7,32 & 4,70 \\
4 & 8,44 & 4,92 \\
5 & 9,87 & 5,65 \\
6 & 9,87 & 6,40 \\
7 & 8,28 & 7,45 \\
8 & 11,14 & 9,64 \\
\hline Média \pm DP & $9,07 \pm 1,20$ & $6,31 \pm 1,67$ \\
\hline
\end{tabular}

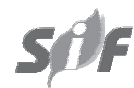

Revista Árvore, Viçosa-MG, v.35, n.4, p.801-807, 2011 
Tabela 2 - Volume com casca (VC) e sem casca (VSC) de madeira e percentual de casca de fustes de Cassia siamea, seccionados por Coccoderus novempunctatus. Seropédica, RJ.2008 .

Table 2 - Volume with bark (VB) and without bark (VWC) of wood and bark percentage of Cassia siamea stems cut by Coccoderus novempunctatus. Seropédica, RJ.2008.

\begin{tabular}{|c|c|c|c|}
\hline Fuste & $\mathrm{VC}\left(\mathrm{m}^{3}\right)$ & $\operatorname{VSC}\left(\mathrm{m}^{3}\right)$ & Casca(\%) \\
\hline 1 & 0,0187 & 0,0162 & 13,37 \\
\hline 2 & 0,0287 & 0,0238 & 17,07 \\
\hline 3 & 0,0094 & 0,0079 & 15,96 \\
\hline 4 & 0,0167 & 0,0134 & 19,76 \\
\hline 5 & 0,0235 & 0,0203 & 13,61 \\
\hline 6 & 0,0330 & 0,0255 & 22,73 \\
\hline 7 & 0,0255 & 0,0216 & 15,29 \\
\hline 8 & 0,0527 & 0,0454 & 13,85 \\
\hline Média \pm DP & $0,0261 \pm 0,0130$ & $0,0218 \pm 0,0112$ & 16,46 \\
\hline Total & 0,2087 & 0,1744 & 16.38 \\
\hline
\end{tabular}

Como relatado anteriormente, trabalhos que avaliam o volume de madeira dos ramos, ou envolvido com cerambicídeos que promovem a queda de fustes ou ramos de espécies florestais são escassos, embora pertinentes, uma vez que servem de subsídio na avaliação trófica dos insetos e danos realizados.

No caso de C. novempunctatus, não há estudo sobre seus danos. Já para o gênero Oncideres sp., Link et al. (1994) avaliaram o volume de madeira consumido por Oncideres saga e Oncideres dejeani em Parapiptadenia rígida. Os dados serviram de base para explicar questões relacionadas à adequação nutricional do hospedeiro ao inseto.

Costa (1986), avaliando a relação do volume de galhos cortados de Mimosa scabrella, seccionados pelo cerambicídeo Oncideres impluviata, concluiu que o corte dos galhos, o número de posturas e o desenvolvimento das larvas são determinados em função do volume de madeira do galho, variável que melhor explicou essas atividades.

As informações obtidas de trabalhos que avaliam os volumes de madeira consumidos e os volumes totais do ramo ou fuste de espécies florestais seccionados por esse grupo de insetos podem fornecer parâmetros que sirvam de auxílio para o cálculo de perdas econômicas ou paisagísticas em plantio de espécies florestais, ou para estudos ecológicos e etológicos dos insetos.

Diante desses resultados, recomenda-se coletar os segmentos da árvore seccionados pela ação da larva e proceder à sua incineração. No intuito de minimizar os danos, sugere-se a poda das brotações emitidas em função da ruptura do fuste ou ramo, escolhendose uma para permanecer e direcionar o crescimento da copa, impedindo, desta forma, o desenvolvimento irregular da copa.

\section{CONCLUSÃO}

Com base nas observações realizadas, pode-se concluir que:

-Coccoderus novempunctatus secciona fustes de Cassia Siamea, promovendo uma lesão em espiral em diversas alturas.

- O volume de madeira envolvida no ciclo de vida deste cerambicídeo é superior ao de outras espécies que possuem o mesmo hábito, indicando, desta forma, um dano superior ao das espécies florestais a que está associado.

- Quanto maior é o DAP da árvore, maior é a altura do ponto onde ocorre a cisão do fuste, pois a larva realiza a lesão em partes com diâmetros menores dimensão.

- A espécie florestal Cassia siamea exsuda uma secreção de cor escura pelo tronco, em função de orifícios construídos pela larva em diversas alturas do tronco.

- A atuação do inseto promove uma formação irregular na copa de Cassia siamea, em função da emissão de brotações próximas ao ponto onde ocorreu a ruptura do fuste. 


\section{REFERÊNCIAS}

BONDAR, G. Insectos daninhos à agricultura. Fascículo III. Pragas das laranjeiras e outras aurantiaceas. São Paulo: Aloizio \& Gallo, 1915. 48p.

COSTA, E. C. Artrópodes associados a Bracatinga (Mimosa scabrella Benth.). 1986. 260f. Tese (Doutorado em Ciências Florestais) - Universidade Federal do Paraná, Curitiba, 1986.

DI IORIO, O. Torneutini (Coleoptera: Cerambycidae) of Argentina. Part 2. Biology of Coccoderus novempunctatus (Germar, 1824), Diploschema rotundicolle (Serville, 1834) and Praxithea derourei (Chabrillac,1857). Gionnale Italiano di Entomologia, v.9, p.3-25, 1998.

FARIDAH, H. I.; van der MAESEN, L. J. G. Plant Resources of South-East Asia. Auxillary plants. Leiden: Backhuys, 1997.

FLECHTMANN, C. A. H. Scolytidae em reflorestamentos com pinheiros tropicais. Piracicaba: IPEF, 1995. 201p.

GOLFARI, L. \& MOOSMAYER, H. Manual de reflorestamento do estado do Rio de Janeiro, governo do estado do Rio de Janeiro. 1980.

LINK, D.; COSTA, E. C.; THUM, A. B. Bionomia comparada dos serradores, oncideres saga saga (Dalman, 1823) e Oncideres dejeani (Thomson, 1868) (Coleoptera: Cerambycidae) em Parapiptadenia rígida. Ciência Florestal, v.4, n.1, p.137-144, 1994.
MACHADO, S. A.; FIGUEIREDO FILHO, A. Dendrometria. Curitiba: Edição do Autor, 2003. 309p.

MONNÉ, M. A. Catalogue of the Neotropical Cerambycidae (Coleoptera) with known host plant - Part II: Subfamily Cerambycinae, Tribes Graciliini to Trachyderini. Publicações Avulsas do Museu Nacional, v.90, p.1-119, 2001.

MONNÉ, M. A. Revisão, análise cladística e biogeografia de Coccoderus Buquet (Coleoptera, Cerambycidae. Revista Brasileira de Entomologia, v.49, n.3, p.369-391, 2005.

SILVA NETO, A. J. et al. Ocorrência e danos de Coccoderus novempunctatus (Germar, 1824) em Anadenanthera sp., Cassia grandis L. f. e Cassia siamea Lam. em seropédica, RJ. In: JORNADA DE INICIAÇÃO CIENTÍFICA DA UFRRJ, 17., 2007, Seropédica. Anais... Seropédica: 2003. CD ROM.

SILVA, A. G. A.; ALMEIDA, D. G. Contribuição ao estudo das coleobrocas. Publicações da Divisão de Defesa Sanitária Vegetal, v.16, p.1-100, 1941.

TREVISAN, H. et al. Avaliação de propriedades físicas e mecânicas da madeira de cinco espécies florestais em função da deterioração em dois ambientes. Revista Árvore, v.31, n.1, p.30-37, 2007.

ZANUNCIO, J. C. et al. Sphallenum tuberosum (Coleopteria: Cerambycidae) em plantas de Eucalyptus spp. no Município de Prado, Bahia. Revista Árvore, v.29, n.2, p.339-343, 2005. 